\title{
HOT OXYGEN ATOMS IN THE UPPER ATMOSPHERES OF VENUS AND MARS
}

\author{
Andrew F. Nagy and Thomas E. Cravens \\ Space Physics Research Laboratory, Department of Atmospheric, Oceanic, and Space Sciences \\ The University of Michigan
}

Abstract. The altitude distribution of the hot oxygen density was calculated for the upper atmospheres of Venus and Mars for solar cycle maximum and minimum conditions, respectively. The calculated values for Venus are in good agreement with the observed values and are about a factor of twenty larger than the calculated values for Mars at the exobase.

\section{Introduction}

The purpose of this brief note is to present the results of calculations of the hot oxygen atom populations in the upper atmospheres of both Venus and Mars. Hot atoms were predicted to be present on both planets over a decade ago (e.g. McElroy, 1972, Sze and McElroy, 1975; Ferrin 1976; Wallis, 1978). A hot hydrogen population has been observed by Lyman $\alpha$ observations from a number of different spacecraft at Venus ( e.g. Anderson, 1976) but not at Mars .The presence of hot oxygen at Venus was confirmed by the $1304 \mathrm{~A}$ measurements of the ultraviolet spectrometer on the Pioneer Venus Orbiter (Nagy et al,1981; Paxton,1983). The initial calculations of the hot oxygen atom population of Venus, published by Nagy et al (1981), used some preliminary ion density data, which were higher than the since established mean values and thus led to relatively high predicted hot oxygen densities. The calculations carried out by McElroy (1982) and Paxton (1983), using more recent input parameters, gave lower densities, in general agreement with the published observed values. We repeated our earlier calculations for Venus in order to show that by simply using the accepted values for the ionospheric densities our model values agree with the observed ones. In this paper, we use the same model to calculate the hot oxygen population expected to be found at Mars, near solar minimum conditions, when the two Phobos spacecraft will reach it in 1989. The terrestrial hot oxygen corona was reviewed and discussed by Yee (1980) and Yee et al.(1980) and will not be addressed in this brief note.

\section{Model Calculations and Results}

The calculations presented here used a two stream model (cf. Chandrasekhar, 1960, Nagy and Banks, 1970) to calculate the equilibrium fluxes and energy distribution of the nonthermal component of atomic oxygen. The results from this two stream model were compared, using the same input parameters, to the results of a numerical transport model, based on moments of the Boltzmann equation, developed by Yee (1980), and were found to be in good agreement (Nagy et al, 1981), thus giving us confidence in our model. Once the energy dependent hot oxygen fluxes are calculated below the exosphere, the densities above the exobase are obtained using Liouville's equation (Chamberlain, 1978).

The various assumptions going into the model were discussed in our original paper (Nagy et al., 1981) and will not be repeated here except for brief comments on the following two items. The main source of hot oxygen atoms is

Copyright 1988 by the American Geophysical Union.

Paper number $8 \mathrm{~L} 6759$.

0094-8276/88/008L-6759\$03.00 the dissociative recombination of $\mathrm{O}_{2}+$ ions. The branching ratios for the dissociative recombination of $\mathrm{O}_{2}^{+}$in the original model were adopted from the work of Rohrbaugh and Nisbet (1973); somewhat different branching ratios were proposed more recently by Paxton (1983). Both sets of branching ratios are presented in Table I and were used in the calculations presented in this paper. We also increased the value used for the cross section of elastic collision between the hot and thermal oxygen atoms to $1.2 \times 10^{-15} \mathrm{~cm}^{-2}$ as suggested by McElroy et al.(1982).

The hot oxygen model values published by us earlier for Venus used some preliminary ionospheric data and led to relatively high values. The model results presented here are for typical solar cycle maximum conditions; the neutral atmosphere values were adopted from the model of Hedin et al.(1983) and the ionospheric parameters were taken from Theis et al. (1980). In order to allow future comparisons/tests of these results the most important input parameter values chosen are shown in Figure 1. The calculated hot oxygen densities for typical solar cycle maximum daytime conditions are presented in Figure 2. The results obtained for both sets of branching ratios, as well as the measured values of Paxton and Stewart (1988) are shown for comparison.

We also calculated, using our model, the expected solar cycle minimum hot oxygen population for Mars. The atmospheric and ionospheric input parameters needed for these calculations were taken from the Viking data base (Nier and McElroy, 1977; Hanson et al., 1977, Chen et al., 1978), except for hydrogen, which came from the Lyman $\alpha$ observations (Anderson,1974) The results of these calculations are shown in Figure 3 along with the cold oxygen and hydrogen densities for comparison. The calculated hot oxygen density near the exobase is about a factor of 20 smaller at Mars than at Venus. In the case of Mars, the effect of the different branching ratios is much smaller than that for Venus (not visible in Figure 3), because of the significantly smaller escape energy.

\section{Discussion}

We calculated the mean daytime density distribution of the hot oxygen corona expected to be found at both Venus and

\section{Input Parameters for Venus Calculations}

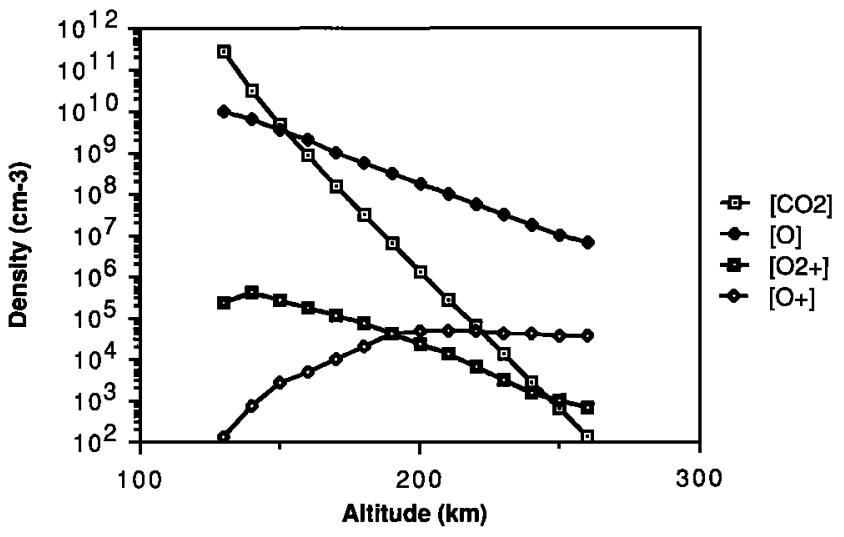

Fig. 1. Major input parameters used in calculating the hot oxygen corona for solar cycle maximum conditions at Venus. 
Table 1. Branching Ratios for the Dissociative Recombination of $\mathrm{O}_{2}+$

\begin{tabular}{cccc}
\hline Products & $\begin{array}{c}\text { Excess Energy } \\
(\mathrm{eV})\end{array}$ & $\begin{array}{c}\text { Rohrbaugh and Nisbet } \\
\text { Values }\end{array}$ & Paxton $^{2}$ Values \\
\hline$O\left({ }^{3} \mathrm{P}\right)+O\left({ }^{3} \mathrm{P}\right)$ & 6.96 & 0.325 & 0.22 \\
$O\left({ }^{3} \mathrm{P}\right)+O\left({ }^{1} \mathrm{D}\right)$ & 5.00 & 0.30 & 0.55 \\
$O\left({ }^{3} \mathrm{P}\right)+O\left({ }^{1} \mathrm{~S}\right)$ & 2.78 & 0.05 & 0.00 \\
$O\left({ }^{1} \mathrm{D}\right)+O\left({ }^{1} \mathrm{D}\right)$ & 3.02 & 0.275 & 0.13 \\
$O\left({ }^{1} \mathrm{D}\right)+O\left({ }^{1}\right)$ & 0.8 & 0.05 & 0.10 \\
\hline
\end{tabular}

${ }^{1}$ Rohrbaugh, R.P. and J.S. Nisbet, Effect of energetic oxygen atoms on neutral density models, J.Geophys. Res, 78 6788, 1973.

2Paxton, L.J., Atomic Carbon in the Venus Thermosphere: Observations and Theory, Ph.D. thesis, University of Colorado, 1983.

Mars. The Venus calculations were carried out for solar cycle maximum conditions, because most of the Pioneer Venus data is from that epoch. On the other hand the Mars calculations correspond to solar cycle minimum conditions because 1) the only appropriate input data base is from the Viking landers, flown in 1976, and 2) the model's most likely near term use will be in association with the Phobos spacecraft measurements which will reach Mars in early 1989. The densities calculated for Venus are about a factor of twenty larger than those obtained for Mars, which is to be expected as the ionospheric densities are also significantly greater on Venus. On Venus, hot oxygen becomes the major neutral gas constituent above about $500 \mathrm{~km}$ on the dayside; only above about $2500 \mathrm{~km}$ does hydrogen become the major neutral species. On the dayside of Mars hot oxygen plays a much less important role, at least during solar cycle minimum conditions (see Figure 3). Even during solar cycle maximum, the hot oxygen population is not expected to dominate at Mars as it will only increase by a factor which is roughly the same as that of the ionizing solar flux. The ionizing EUV solar flux at Mars during solar minimum is approximately a factor of ten less than the solar flux at Venus during solar cycle maximum. However there is a further factor which reduces the hot oxygen densities at Mars; namely the fact that the escape

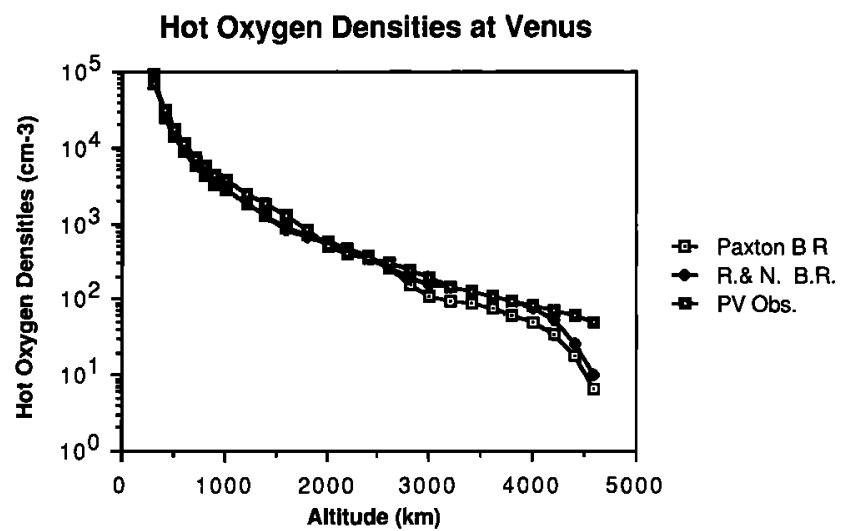

Fig. 2. Measured and calculated hot oxygen densities at Venus. energy at Mars is approximately a quarter of what it is at Venus and this difference accounts for the other factor of about two in the calculated densities. Ip (1989), in a paper recently submitted for publication,presents an excellent discussion of the potential importance and implications of a hot oxygen corona at Mars ; he calculated the distribution function using a Monte Carlo technique and obtained a hot oxygen population about a factor of five greater than the one presented here.

An extended neutral corona about a planetary body has a major impact on the interaction of the solar wind with that body (e.g. Russell et al., 1983, Breus, 1986). This effect is most significant if the neutral gas constituent is "heavy" (heavier than hydrogen) and extends to "great" (in terms of planetary radii) distances. The most extended neutral corona in the solar system are the ones associated with active comets. The solar wind interaction region extends millions of kilometers upstream of active comets such as $\mathrm{P} / \mathrm{Halley}$ (e.g. Somogyi et al., 1986). The knowledge that a hot hydrogen and oxygen corona exists around Venus has lead to number of studies of their effect on the solar wind interaction with Venus (e.g. Gombosi et al.; 1980, Wallis, 1982; Breus, 1986;

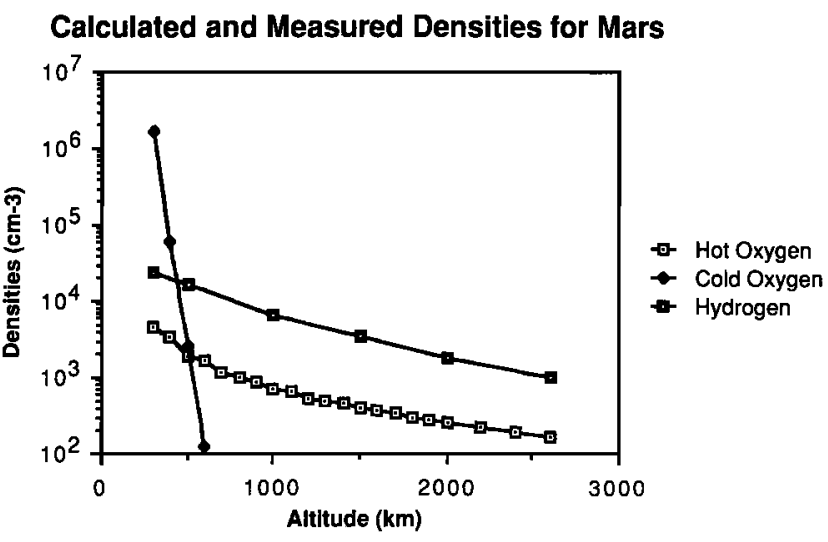

Fig. 3. Hot oxygen densities calculated for solar cycle minimum conditions at Mars; cold oxygen and hydrogen values deduced from observations are also shown for comparison. 
Belotserkovskii et al., 1987), which have established that photoionization and/or charge exchange of the hot oxygen atoms inside the magnetosheath is significant. There is also experimental evidence that these processes are operating in the magnetosheath and that they play an important role in the interaction processes (e.g. Mihalov and Bames, 1981). Mars and Venus are very similar in many ways; therefore, the question of the importance of the hot oxygen corona at Mars has also been raised. However the expected densities, at least during solar cycle minimum, are too low to affect the solar wind interaction processes to any significant degree (Russell et al., 1983; Breus, 1986).

Acknowledoments. The work presented in this paper was carried out with the support of NASA Grants NGR 23-005015, NAG 2-491 and NAGW-15.

\section{References}

Anderson, D.E., Jr., The Mariner 5 ultraviolet photometer experiment: Analysis of hydrogen Lyman alpha data, $\mathbf{I}$ Geophys. Res., 81, 1213, 1976.

Anderson, D. E., Mariner 6, 7, and 9 ultraviolet spectrometer experiment: Analysis of Hydrogen Lyman alpha data, $\underline{\mathbf{J}}$ Geophys. Res. 79, 1513, 1974.

Belotserkovskii, O.M., T.K. Breus, A.M. Krymskii, V. Ya Mitnitskii, A.F. Nagy and T.I. Gombosi, The effect of the hot oxygen corona on the interaction of the solar wind with Venus, Geophys. Res. Lett., 14, 503, 1987.

Breus, T. K., Mass loading of Venus: Theoretical expectations, Adv. Space Res., 6, 167, 1986.

Chamberlain, J.W., Theory of Planetary Atmospheres, Academic Press, New York, 1978.

Chandrasekhar, S., Radiative Transfer, Dover, New York, 1960.

Chen, R. H., T. E. Cravens and A. F. Nagy, The Martian ionosphere in light of the Viking observations, J. Geophys. Res. 83, 3871, 1978.

Ferrin, I.R., The distribution of hot hydrogen atoms in the upper atmosphere of Venus, Ph.D. Thesis, University of Colorado, Boulder, 1976

Gombosi T. I., T. E. Cravens, A. F. Nagy, R. C. Elphic and C. T. Russell, Solar wind absorption by Venus, J. Geophys. Res. $185,7747,1980$.

Hanson, W. B., S Sanatani and D. R. Zuccaro, The Martian ionosphere as observed by the Viking retarding potential analyzers, J. Geophys. Res. 82, 4351, 1977.

Hedin, A. E., H. B. Niemann, W. T. Kasprzak and A. Seiff, Global empirical model of the Venus thermosphere, J. Geophys. Res. 88. 73, 1983.

Ip, W. H., On a hot oxygen corona of Mars, submitted to Icarus, 1988.

Luhmann, J. G., The solar wind interaction with Venus, Space Sci. Rev. 44, 241, 1986.

McElroy, M.B., Mars: An evolving atmosphere, Science, $175,443,1972$.
McElroy, M. B., M. J. Prather and J. M. Rodriguez, Loss of oxygen from Venus, Geophys. Res. Lett. 2, 649, 1982

Mihalov, J. D. and A. Barnes, Evidence for the acceleration of ionospheric $\mathrm{O}^{+}$in the magnetosheath of Venus, Geophys. Res. Lett. 8, 1277, 1981 .

Nagy, A.F. and P.M. Banks, Photoelectron fluxes in the ionosphere, J. Geophys. Res., 75, 6260, 1970.

Nagy, A.F., T.E. Cravens, J.H. Yee and A.I.P. Stewart, Hot oxygen atoms in the upper atmosphere of Venus, Geophys. Res. Lett. 8, 629, 1981.

Nier, A. O., and M. B. McElroy, Composition and structure of Mars' upper atmosphere: Results from the neutral mass spectrometers on Viking 1 and 2, L. Geophys. Res. 82, 4341, 1977.

Paxton, L.J., Atomic Carbon in the Venus Thermosphere: Observations and Theory, Ph. D. Thesis, University of Colorado, 1983.

Paxton, L.J., and A. I. Stewart, The hot oxygen corona of Venus, to be submitted J. Geophys, Res, 1988.

Rohrbaugh, R.P. and J.S. Nisbet, Effect of energetic oxygen atoms on neutral density models, J. Geophys. Res., 78, 6788, 1973.

Russell, C. T., T. I. Gombosi, M. Horanyi, T. E. Cravens and A. F. Nagy, Charge exchange in the magnetosheath of Venus and Mars: A comparison, Geophys. Res. Lett. 10, $163,1983$.

Somogyi A. J. et al., First observations of energetic particles near comet Halley, Nature. 321, 285, 1986.

Sze, N.D. and M.B. McElroy, Some problems in Venus aeronomy, Planet. Space Sci. 23, 763, 1975.

Theis, R. F., L. H. Brace and H. G. Mayr, Empirical models of the electron temperature and density in the Venus ionosphere, J. Geophys. Res. 85, 7787, 1980.

Wallis, M.K.,Exospheric density and escape fluxes of atomic isotopes on Venus and Mars, Planet..Space Sci., 26, 949, 1978.

Wallis, M.K., Comet like interaction of Venus with the solar wind III: The atomic oxygen corona, Geophys. Res. Lett., 9, 427, 1982.

Yee, J.-H., Theoretical and experimental study on the atomic oxygen corona in the earth's atmosphere, Ph.D. Thesis, The University of Michigan, Ann Arbor, 1980.

Yee, J. H., J. W. Meriwether, and P. B. Hays, Detection of a corona of fast oxygen atoms during solar maximum, $\mathbf{J}$. Geophys. Res.. $\underline{85}, 3396,1980$.

A.F. Nagy and T. E. Cravens, The University of Michigan, Space Physics Research Laboratory, Department of Atmospheric, Oceanic, and Space Sciences, Ann Arbor, Michigan 48109-2143.

(Received February 5, 1988; revised March 15, 1988; accepted April 12, 1988.) 